\title{
Importância do rastreamento do câncer colorretal: uma revisão
}

Importance of colorectal cancer screening: a review

Importancia de la detección del cáncer colorrectal: una revisión

Recebido: 03/10/2021 | Revisado: 10/10/2021 | Aceito: 16/10/2021 | Publicado: 19/10/2021

Lennara Pereira Mota

ORCID: https://orcid.org/0000-0002-2629-6634 Centro Universitário Maurício de Nassau, Brasil

E-mail: lennaramota@hotmail.com

Maria Vitalina Alves de Sousa

ORCID: https://orcid.org/0000-0003-4448-2489

Centro Universitário INTA, Brasil

E-mail: enf.vitalinaalves@gmail.com

Amanda Eckhardt

ORCID: https://orcid.org/0000-0002-9733-9689

Centro Universitário INTA, Brasil

E-mail: amanda_e2008@hotmail.com

Maria Santana do Nascimento

ORCID: https://orcid.org/0000-0003-0527-4636

Centro Universitário INTA, Brasil

E-mail: msantanamsn@gmail.com

Lyrlanda Maria Cavalcante de Almeida

ORCID: https://orcid.org/0000-0003-0390-0090

Centro Universitário INTA, Brasil

E-mail: lyrlanda97@gmail.com

Juliana Maria de Freitas

ORCID: https://orcid.org/0000-0002-0651-3371

Centro Universitário INTA, Brasil

E-mail: julianafhz@gmail.com

Alexandra Rodrigues Cardoso

ORCID: https://orcid.org/0000-0002-7277-4668

Centro Universitário Maurício de Nassau, Brasil

E-mail: alexandrarodcar@gmail.com

João Pedro Tavares De Oliveira

ORCID: https://orcid.org/0000-0001-6887-1812

Centro Universitário Uninovafapi, Brasil

E-mail: Jpto30@hotmail.com

Joelma Maria dos Santos da Silva Apolinário

ORCID: https://orcid.org/0000-0001-9521-9432

Centro Universitário Maurício de Nassau, Brasil

E-mail: jo.silva00@hotmail.com

Francisco Wagner dos Santos Sousa

ORCID: https://orcid.org/0000-0001-9309-2925

Universidade Estadual do Piauí, Brasil

E-mail: wagnersantosreal@gmail.com

Amanda Oliveira Leão

ORCID https://orcid.org/0000-0001-9272-0379

Centro Universitário Maurício de Nassau, Brasil

E-mail: amandaoleao96@gmail.com

Ana Alinne Gomes Da Penha

ORCID: https://orcid.org/0000-0001-9253-1199

Universidade Regional Do Cariri, Brasil

E-mail: anaalinne.nurse@gmail.com

Eldson Rodrigues Borges

ORCID: https://orcid.org/0000-0002-8848-0074

Centro Universitário Uninovafapi, Brasil

E-mail: eldsonborges7@gmail.com

Emanuelle da Costa Gomes

ORCID: https://orcid.org/0000-0003-3672-7108

Universidade Estadual do Piauí, Brasil

E-mail: emanuellecg48@gmail.com

Kacilia Bastos de Castro Rodrigues

ORCID: https://orcid.org/0000-0003-0068-2390

Faculdade Aliança, Brasil

E-mail: kaciliacastro@hotmail.com 


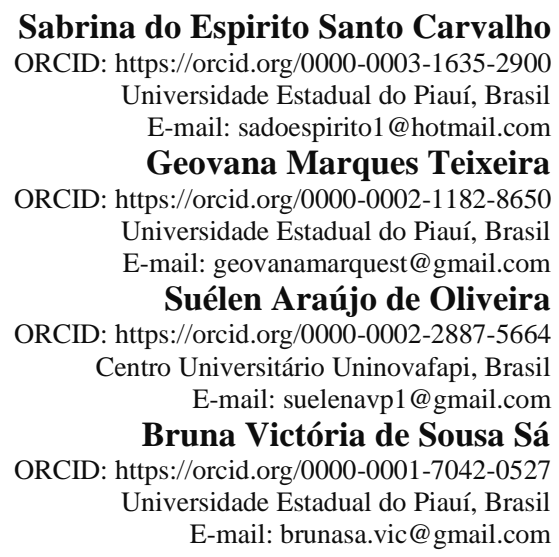

\title{
Resumo
}

No mundo, o câncer colorretal é considerado o mais fatal e a segunda principal causa de morte por câncer. Fatores como envelhecimento da população e hábitos de vida possuem relação com a prevalência deste câncer. Este artigo de revisão tem por objetivo expor por meio da análise de artigos científicos a importância do diagnóstico precoce em pacientes com fatores de risco para o câncer colorretal. Trata-se de uma revisão bibliográfica de caráter qualitativo sistemático realizado entre os anos de 2018 a 2021 nos idiomas português, inglês e espanhol. Dados epidemiológicos reforçam a importância do rastreamento do câncer, principalmente devido ao aumento de casos de CCR nos últimos 30 anos. Esta realidade é atribuída a fatores ambientais, principalmente relacionados ao estilo de vida (sedentarismo, desnutrição, obesidade, tabagismo) e envelhecimento da população. Portanto, a triagem é baseada nos fatores de risco. O rastreio deve iniciar aos 45 anos, pois a detecção precoce do CCR pode reduzir significativamente as taxas de mortalidade. O câncer colorretal é considerado uma das neoplasias mais prevalentes do mundo. Anteriormente era mais comum em pacientes com idade acima de 50 anos, mas essa realidade tem mudado devido ao estilo de vida da população. Infelizmente não há um rastreio desse tipo de câncer que seja oferecido pelo Sistema Único de Saúde (SUS) e por isso o diagnóstico precoce não ocorre com a frequência esperada. É necessário que sejam desenvolvidas formas de rastreio deste câncer, assim como as realizadas no câncer de mama e de próstata, visto que o câncer colorretal é agressivo e possui altas taxas de mortalidade. Palavras-chave: Neoplasia colorretal; Avaliação de risco; Mortalidade; Qualidade de vida.

\begin{abstract}
Worldwide, colorectal cancer is considered the most fatal and the second leading cause of cancer death. Factors such as population aging and lifestyle habits are related to the prevalence of this cancer. This review article aims to expose, through the analysis of scientific articles, the importance of early diagnosis in patients with risk factors for colorectal cancer. This is a systematic qualitative bibliographic review carried out between the years 2018 to 2021 in Portuguese, English and Spanish. Epidemiological data reinforce the importance of cancer screening, mainly due to the increase in RCC cases in the last 30 years. This reality is attributed to environmental factors, mainly related to lifestyle (sedentary lifestyle, malnutrition, obesity, smoking) and population aging. Therefore, screening is based on risk factors. Screening should start at age 45, as early detection of CCR can significantly reduce mortality rates. Colorectal cancer is considered one of the most prevalent neoplasms in the world. Previously it was more common in patients over the age of 50 years, but this reality has changed due to the lifestyle of the population. Unfortunately, there is no screening for this type of cancer that is offered by the Unified Health System (SUS) and, therefore, early diagnosis does not occur as often as expected. . It is necessary to develop forms of screening for this cancer, as well as those carried out in breast and prostate cancer, since colorectal cancer is aggressive and has high mortality rates.
\end{abstract}

Keywords: Colorectal neoplasm; Risk assessment; Mortality; Quality of life.

\section{Resumen}

En todo el mundo, el cáncer colorrectal se considera el más mortal y la segunda causa principal de muerte por cáncer. Factores como el envejecimiento de la población y los hábitos de vida están relacionados con la prevalencia de este cáncer. Este artículo de revisión tiene como objetivo exponer, a través del análisis de artículos científicos, la importancia del diagnóstico precoz en pacientes con factores de riesgo de cáncer colorrectal. Se trata de una revisión bibliográfica cualitativa sistemática realizada entre los años 2018 a 2021 en portugués, inglés y español. Los datos epidemiológicos refuerzan la importancia del cribado del cáncer, principalmente debido al aumento de casos de CCR en los últimos 30 años. Esta realidad se atribuye a factores ambientales, principalmente relacionados con el estilo de vida (sedentarismo, desnutrición, obesidad, tabaquismo) y el envejecimiento de la población. Por lo tanto, el cribado se basa en factores de riesgo. La detección debe comenzar a los 45 años, ya que la detección temprana de CCR puede reducir significativamente las tasas de mortalidad. El cáncer colorrectal se considera una de las neoplasias más prevalentes en el mundo. Anteriormente era más común en pacientes mayores de 50 años, pero esta realidad ha cambiado debido al estilo de vida de la población. Desafortunadamente, no existe una prueba de detección para este tipo de cáncer que ofrece el 
Sistema Único de Salud (SUS) y, por lo tanto, el diagnóstico temprano no ocurre con la frecuencia esperada. . Es necesario desarrollar formas de cribado de este cáncer, así como los que se realizan en el cáncer de mama y de próstata, ya que el cáncer colorrectal es agresivo y tiene altas tasas de mortalidad.

Palabras clave: Neoplasia colorrectal; Evaluación de riesgos; Mortalidad; Calidad de vida.

\section{Introdução}

O câncer colorretal é considerado uma das neoplasias mais comuns mundialmente, sendo bastante prevalente no Brasil. De acordo com os dados da Agência Internacional de Pesquisa sobre Câncer, em 2018 foi estimado que o câncer colorretal possa aumentar cerca de $75 \%$ até o ano de 2040, com mais de 11,4 milhões de novos casos e mais de 6,1 milhões de óbitos. No mundo, o câncer colorretal é considerado o mais fatal e a segunda principal causa de morte por câncer. Cerca de 900.000 pessoas morrem todos os anos. Fatores como envelhecimento da população e hábitos de vida possuem relação com a prevalência deste câncer. Maus hábitos alimentares, obesidade, carência de exercício físico e o tabagismo aumentam o risco de doença (Bray et al., 2018; Keum; Giovannucci, 2019; Macêdo et al., 2020; Moura et al.,2020).

Segundo estudo realizado com pacientes atendidos no Instituto Nacional do Câncer (INCA), os sintomas mais comuns foram listados em ordem decrescente: emagrecimento, dor, boca seca e preocupação. Por outro lado, os menos comuns são tosse, disfunção urinária, disfagia e úlceras orais. Os sintomas de câncer colorreral (CCR) também podem variar dependendo da faixa etária afetada. Pacientes jovens têm mais sintomas físicos e o impacto social da doença, enquanto pessoas mais idosas têm mais sintomas psicológicos (Mello et al., 2020).

De acordo com o estadiamento dos tumores colorretais, o principal método de tratamento é a cirurgia, portanto, a cirurgia mais completa envolve a retirada do intestino grosso e do reto. É necessária a realização de uma estomia, que possui impacto na sociedade, no físico e no psicológico dos pacientes. A estomia é uma abertura realizada principalmente no trato gastrointestinal, e seus tipos principais são uma abertura com a função de eliminação intestinal, denominada colostomias e ileostomias. Este procedimento cirúrgico visa evitar complicações causadas por tumores e doenças intestinais (Macêdo et al., 2020).

O câncer de intestino envolve tumores que acometem partes do intestino grosso, chamadas cólon, reto e ânus. Os fatores de risco do CCR estão relacionados à suscetibilidade genética, doenças inflamatórias e ao impacto do estilo de vida, especialmente dieta inadequada, estilo de vida sedentário, fumo, bebida excessiva e grande ingestão de carnes processadas e / ou vermelhas, hábitos que variam geralmente de acordo com as condições socioeconômicas (Silva et al., 2021).

Para o diagnóstico de CCR, pode-se realizar proctoscopia, incluindo anuscopia e colonoscopia retossigmóide, permitindo o estudo da mucosa do cólon retal e sigmóide e de possíveis lesões suspeitas. Se esta avaliação não for suficiente, é realizado a colonoscopia que permite a biópsia de lesões que não podem ser alcançadas pela colonoscopia retossigmóide (Paes et al., 2021).

Estudos descrevem um aumento da incidência de câncer colorretal entre jovens nas últimas décadas. Esse aumento tem sido atribuído ao rastreamento inadequado e ao aumento dos fatores de risco relacionados à obesidade e à dieta alimentar (Moura et al.,2020; Silva et al., 2020).

O câncer colorretal é uma das neoplasias mais prevalentes do mundo com uma alta taxa de mortalidade. Sabe-se que geralmente esta doença apresenta-se assintomática, sendo necessário o rastreamento em pacientes com fatores de risco para que seja realizado o tratamento o mais rápido possível, aumentando as chances de cura e a qualidade de vida dos pacientes. Este artigo de revisão tem por objetivo expor por meio da análise de artigos científicos a importância do diagnóstico precoce em pacientes com fatores de risco para o câncer colorretal. 


\section{Métodos}

Trata-se de uma revisão bibliográfica de caráter qualitativo sistemático. A busca pelos textos foi realizada a partir das seguintes palavras-chaves indexadas no DECS (Descritores em Ciências da Saúde): "Neoplasia colorretal”, “Avaliação de risco", "Mortalidade" e "Qualidade de vida" nos idiomas português, inglês e espanhol na plataforma Scientific Electronic Library Online (SCIELO) e Instituto Nacional do Câncer (INCA) - Revista Brasileira de Cancerologia.

Os critérios de inclusão foram pesquisas científicas publicadas de 2018 a 2021, buscando informações atualizadas a cerca do tema, publicados no idioma português, inglês e espanhol, que atendiam ao problema da pesquisa: Qual a importância do rastreamento do câncer colorretal em pacientes que apresentam fatores de risco para esta neoplasia? Os critérios de exclusão foram trabalhos científicos publicados com data anterior ao ano de 2018, com apenas resumos disponíveis, publicações duplicadas, artigos de relato de experiência, reflexivo, editoriais, comentários e cartas ao editor.

A partir do problema de pesquisa foram selecionados artigos publicados em periódicos nacionais escolhidos a partir de levantamento realizado por meios dos descritores na biblioteca virtual Scientific Electronic Library Online e Instituto Nacional do Câncer (INCA) - Revista Brasileira de Cancerologia e dissertações e teses.

Os artigos foram selecionados para leitura e foram incluídos na análise qualitativa artigos que se enquadravam no tema proposto do trabalho.

\section{Resultados e Discussão}

A Figura 1 ilustra os resultados deste trabalho que foram obtidos através da análise qualitativa de publicações sobre o rastreamento do câncer colorretal em pacientes com fatores de risco nas bases de dados Scientific Electronic Library Online e Instituto Nacional do Câncer (INCA) - Revista Brasileira de Cancerologia. 
Figura 1: Fluxograma que apresenta o processo de seleção das publicações sobre neoplasia colorretal e o rastreamento em pacientes com fatores de risco, Teresina, Brasil.
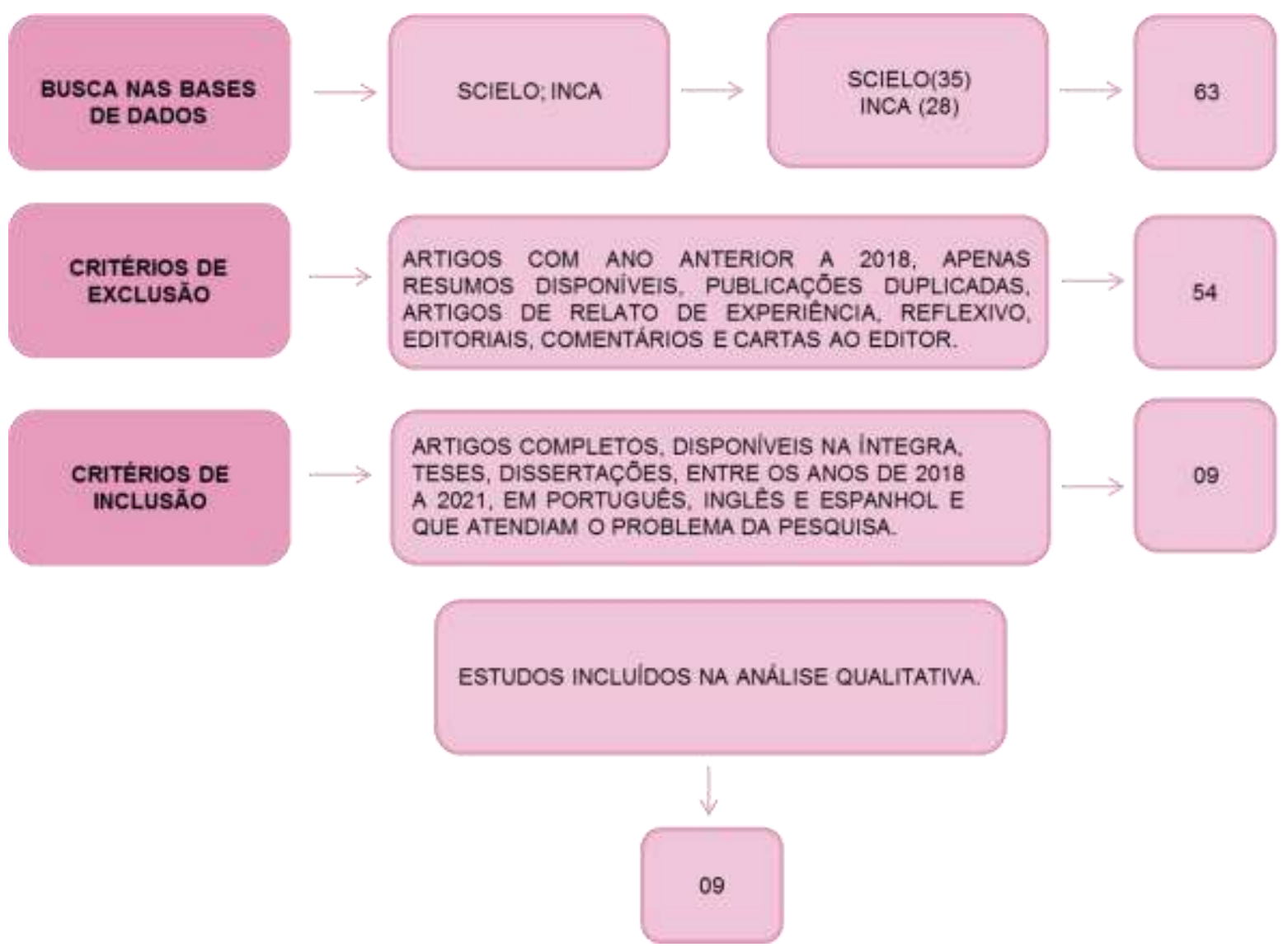

Fonte: Mota et al. (2021).

Os resultados deste trabalho foram obtidos através da análise qualitativa de publicações sobre o rastreamento do câncer colorretal em pacientes com fatores de risco encontrados nas bases de dados Scientific Electronic Library Online e Instituto Nacional do Câncer (INCA) - Revista Brasileira de Cancerologia obtendo 35 artigos na Scielo e 28 artigos no INCA. Foram excluídos 30 artigos da Scielo e 24 do INCA por não se enquadrarem no tema proposto. Foram incluídos na análise qualitativa 05 artigos da Scielo e 04 artigos da INCA entre os anos de 2018 a 2021.

De acordo com a pesquisa realizada nas bases de dados, foi possivel listar os principais títulos e objetivos dos estudos publicados na literatura, utilizados neste trabalho conforme o Quadro 1. 
Quadro 1: Principais títulos e objetivos dos artigos utilizados na pesquisa, Teresina, Brasil, 2021.

\begin{tabular}{|c|c|c|}
\hline ANO DE PUBLICAÇÃO & TÍTULO & OBJETIVO \\
\hline Moura et al.,2020. & $\begin{array}{c}\text { Padrão Sintomatológico em } \\
\text { Pacientes do Câncer Colorretal de } \\
\text { acordo com a Idade. }\end{array}$ & $\begin{array}{l}\text { Investigar a diferença no padrão de } \\
\text { sintomas entre pacientes adultos e idosos } \\
\text { com câncer de cólon e reto. }\end{array}$ \\
\hline Macêdo et al., 2020. & $\begin{array}{l}\text { Percepções de pacientes } \\
\text { estomizados com câncer colorretal } \\
\text { acerca da qualidade de vida. }\end{array}$ & $\begin{array}{l}\text { Compreender as percepções de pacientes } \\
\text { afetados por neoplasia colorretal com } \\
\text { estomias acerca da qualidade de vida. }\end{array}$ \\
\hline Palmeira et al., 2020. & $\begin{array}{l}\text { Evolução comparativa e temporal } \\
\text { das tendências de mortalidade por } \\
\text { Câncer Colorretal em Sergipe e } \\
\text { Nordeste no período de } 2008 \text { a } \\
2018 . \\
\end{array}$ & $\begin{array}{l}\text { Analisar a evolução temporal e comparar } \\
\text { as tendências de taxa de mortalidade por } \\
\text { CCR no estado de Sergipe e no Nordeste } \\
\text { brasileiro, no período de } 2008 \text { a } 2018 .\end{array}$ \\
\hline De Paula Pires et al., 2021. & $\begin{array}{l}\text { Rastreamento do Câncer Colorretal: } \\
\text { Revisão de literatura. }\end{array}$ & $\begin{array}{l}\text { Realizar revisão de literatura a respeito } \\
\text { do rastreamento de câncer colorretal } \\
\text { entre os anos de 2014-2019. }\end{array}$ \\
\hline Paes et al., 2021. & $\begin{array}{l}\text { Análise das complicações pós- } \\
\text { operatórias de câncer colorretal. }\end{array}$ & $\begin{array}{l}\text { Esse estudo, através de uma revisão } \\
\text { narrativa, objetiva determinar as } \\
\text { possíveis complicações pós-operatórias } \\
\text { do Câncer Colorretal (CCR), um dos } 3 \\
\text { tumores mais prevalentes no mundo, } \\
\text { baseado na abordagem escolhida. }\end{array}$ \\
\hline Zanatto et al., 2020. & $\begin{array}{c}\text { Impacto das mutações kras e } \\
\text { características clínicas em câncer } \\
\text { colorretal. }\end{array}$ & $\begin{array}{l}\text { Investigar a associação de características } \\
\text { clinicopatológicas com mutações no } \\
\text { KRAS em pacientes com câncer } \\
\text { colorretal tratados. } \\
\end{array}$ \\
\hline Aguiar et al., 2020. & $\begin{array}{l}\text { Sobrevida de pacientes com câncer } \\
\text { colorretal em um Centro de Câncer. }\end{array}$ & $\begin{array}{l}\text { Descrever a sobrevida global e analisar } \\
\text { os fatores prognósticos de pacientes } \\
\text { tratados por câncer colorretal em um } \\
\text { centro de oncologia. }\end{array}$ \\
\hline Silva et al., 2020. & $\begin{array}{c}\text { Câncer colorretal em pacientes com } \\
\text { idade inferior a } 50 \text { anos - } \\
\text { experiência em cinco anos. }\end{array}$ & $\begin{array}{c}\text { Avaliar o perfil clínico de pacientes } \\
\text { portadores de câncer colorretal com idade } \\
\text { inferior a } 50 \text { anos atendidos em um } \\
\text { hospital público de Brasília ao longo de } 5 \\
\text { anos. }\end{array}$ \\
\hline Puty et al., 2020. & $\begin{array}{l}\text { Perfil da qualidade de vida de } \\
\text { pacientes com câncer colorretal } \\
\text { estádios III e IV em instituição } \\
\text { privada no Estado do Pará: Estudo } \\
\text { seccional. }\end{array}$ & $\begin{array}{l}\text { Descrever a qualidade de vida de } \\
\text { pacientes com câncer colorretal estágios } \\
\text { III e IV em uma instituição no estado do } \\
\text { Pará. }\end{array}$ \\
\hline
\end{tabular}

Fonte: SCIELO e INCA.

Desta forma, foi possível listar os principais resultados e conclusões dos estudos publicados na literatura, conforme o

Quadro 2.

Quadro 2: Resultados e conclusões dos artigos utilizados, Teresina, Brasil, 2021.

\begin{tabular}{|c|c|c|}
\hline PUBLICAÇÃO & RESULTADOS & CONCLUSÕES \\
\hline Moura et al.,2020. & $\begin{array}{c}\text { De forma geral, para a maioria dos sintomas, } \\
\text { não houve diferença na ocorrência entre os } \\
\text { mais novos e os mais velhos. Entretanto, houve } \\
\text { significância estatística para dor }(\mathrm{p}=0,033), \\
\text { nervosismo }(\mathrm{p}=0,013) \text {, sonolência }(\mathrm{p}=0,033), \\
\text { tristeza }(\mathrm{p}=0,003) \text {, problemas com desejo } \\
\text { sexual ou atividade sexual }(\mathrm{p}=0,014) \text {, falta de } \\
\text { apetite ( } \mathrm{p}=0,028) \text {, irritação }(\mathrm{p}=0,013) \text {, mudança } \\
\text { nos gostos dos alimentos }(\mathrm{p}=0,042), \text { perda de } \\
\text { cabelo }(\mathrm{p}=0,002) .\end{array}$ & $\begin{array}{l}\text { A carga sintomatológica frente ao } \\
\text { câncer colorretal pode apresentar } \\
\text { distinção conforme a idade. Isso é } \\
\text { relevante, pois reforça a ideia de } \\
\text { individualizar o tratamento para } \\
\text { melhorar a assistência e, } \\
\text { consequentemente, a qualidade de vida } \\
\text { desses doentes. }\end{array}$ \\
\hline Macêdo et al., 2020. & $\begin{array}{c}\text { Emergiram três categorias temáticas: Qualidade } \\
\text { de vida: influências sociais, psíquicas e } \\
\text { espirituais; Adaptações pessoais e ambientais } \\
\text { frente à nova realidade e Complicações no } \\
\text { viver com estomia. }\end{array}$ & $\begin{array}{l}\text { Pacientes com estomias proveniente de } \\
\text { câncer colorretal, em geral, têm } \\
\text { variações nas percepções de qualidade } \\
\text { de vida, de forma que, no domínio } \\
\text { físico, consideraram o dispositivo vital } \\
\text { no tratamento, mas que gerou, a priori, }\end{array}$ \\
\hline
\end{tabular}




\begin{tabular}{|c|c|c|}
\hline & & $\begin{array}{l}\text { isolamento social, por vergonha, } \\
\text { necessitando de adaptações diante da } \\
\text { nova realidade, a fim de reduzir o } \\
\text { impacto emocional, muito } \\
\text { frequentemente representado em } \\
\text { forma de tristeza e não aceitação. }\end{array}$ \\
\hline Palmeira et al., 2020. & $\begin{array}{l}\text { Os óbitos por CCR em Sergipe e no Nordeste, } \\
\text { foi predominante na região do cólon, seguido } \\
\text { pelo reto e junção retossigmoide, prevaleceu } \\
\text { nos idosos, com semelhança entre os sexos, } \\
\text { porém ligeiramente maior nas mulheres. }\end{array}$ & $\begin{array}{c}\text { Necessita-se do fortalecimento e } \\
\text { ampliação de medidas de promoção } \\
\text { em saúde com ações preventivas, de } \\
\text { diagnóstico precoce e de tratamento do } \\
\text { CCR focadas no rastreamento da } \\
\text { população, principalmente a mais } \\
\text { idosa. }\end{array}$ \\
\hline De Paula Pires et al., 2021. & $\begin{array}{l}\text { O rastreamento é preconizado a partir dos } 45 \\
\text { anos de idade e os métodos podem ser: } \\
\text { Pesquisa de sangue oculto nas fezes (Teste } \\
\text { Imunoquímico Fecal, Guaiaco, DNA fecal) } \\
\text { Colonoscopia, Sigmoidoscopia, Colonografia } \\
\text { Tomográfica Computadorizada e Micro-RNAs. }\end{array}$ & $\begin{array}{c}\text { Evidencia-se a importância de } \\
\text { métodos diagnósticos seguros e que } \\
\text { apresentem custo-benefício satisfatório } \\
\text { para melhor adesão ao rastreamento de } \\
\text { CCR, objetivando tratamento precoce } \\
\text { e diminuição da mortalidade em } \\
\text { decorrência da doença. } \\
\end{array}$ \\
\hline Paes et al., 2021. & $\begin{array}{c}\text { Apesar de todos os procedimentos cirúrgicos } \\
\text { para tratamento de CCR conferirem riscos de } \\
\text { complicações, intervenções de urgência e } \\
\text { cirurgias abertas apresentam maiores taxas } \\
\text { quando comparados a cirurgias minimamente } \\
\text { invasivas. }\end{array}$ & $\begin{array}{l}\text { A cirurgia de escolha dependerá de } \\
\text { diversos fatores incluindo eficiência e } \\
\text { eficácia do método para cada estágio } \\
\text { da doença, experiência do cirurgião e } \\
\text { fatores de risco do paciente, sempre } \\
\text { visando a menor probabilidade de } \\
\text { complicações. }\end{array}$ \\
\hline Zanatto et al., 2020. & $\begin{array}{l}\text { A mutação KRAS foi identificada em 43,4\% } \\
\text { dos pacientes, c.35G }>\text { T (p.G12V), c.35G }>\text { A } \\
\text { (p.G12D) e c.38G }>\text { A (p.G13D). Não foi } \\
\text { encontrada correlação entre a mutação KRAS e } \\
\text { a idade ( } \mathrm{p}=0,646 \text { ) ou o gênero (p=0,815). No } \\
\text { entanto, o grupo mutado apresentou níveis mais } \\
\text { altos de CEA na admissão ( } \mathrm{p}=0,048) \text {. }\end{array}$ & $\begin{array}{c}\text { A mutação KRAS foi identificada em } \\
\text { quase metade dos pacientes. O grupo } \\
\text { KRAS mutado apresentou níveis mais } \\
\text { altos de CEA na admissão e a mutação } \\
\text { no códon } 13 \text { foi associada ao } \\
\text { envolvimento de mais de um local } \\
\text { metastático no curso da doença. A } \\
\text { doença do cólon foi associada com } \\
\text { pior sobrevida global. }\end{array}$ \\
\hline Aguiar et al., 2020. & $\begin{array}{l}\text { Dos } 2.279 \text { casos de câncer colorretal } \\
\text { analisados, } 58,4 \% \text { estavam no cólon. A taxa de } \\
\text { sobrevida global em } 5 \text { anos para pacientes com } \\
\text { câncer colorretal foi de } 63,5 \% \text { ( } 65,6 \% \text { e } 60,6 \% \\
\text { para neoplasias colônicas e retais, } \\
\text { respectivamente). }\end{array}$ & $\begin{array}{l}\text { A melhor sobrevida de pacientes com } \\
\text { câncer colorretal melhora com o } \\
\text { estágio inicial e o tratamento é } \\
\text { iniciado dentro de } 60 \text { dias após o } \\
\text { diagnóstico. A idade acima de } 70 \text { anos } \\
\text { foi um fator independente preditivo de } \\
\text { mau prognóstico. A sobrevida global } \\
\text { aumentou para todos os pacientes } \\
\text { tratados no período de } 2000-2004 \text { a } \\
\text { 2010-2013. }\end{array}$ \\
\hline Silva et al., 2020. & $\begin{array}{c}\text { No grupo dos pacientes com idade inferior a } 50 \\
\text { anos houve maior concentração de indivíduos } \\
\text { com estadiamento III e IV ( } \mathrm{p}=0,041) \text {, foi mais } \\
\text { frequente a presença de tumores pouco } \\
\text { diferenciados }(10,25 \% \text { contra } 3,52 \% ; \mathrm{p}=0,153), \\
\text { foram descritas maiores incidências de margens } \\
\text { cirúrgicas comprometidas ( } \mathrm{p}=0,368) \text {, invasão } \\
\text { angiolinfática ( } \mathrm{p}=0,07 \text { ) e perineural ( } \mathrm{p}=0,007), \\
\text { o que denota doença mais avançada nesse } \\
\text { grupo de pacientes. }\end{array}$ & $\begin{array}{l}\text { O estudo evidenciou a baixa } \\
\text { efetividade dos métodos de } \\
\text { rastreamento populacional para câncer } \\
\text { colorretal atualmente empregados } \\
\text { nesta população, visto a elevada } \\
\text { incidência da doença e ao diagnóstico } \\
\text { tardio em ambos os grupos. }\end{array}$ \\
\hline Puty et al., 2020. & $\begin{array}{c}\text { A média de idade foi de } 57,86 \text { anos }(\mathrm{dp}= \\
\text { 14,58). Considerando a média de qualidade de } \\
\text { vida, avaliada pelo SF-36, a limitação por } \\
\text { aspectos físicos foi a categoria com maiores } \\
\text { extremos de respostas (mediana: } 37,5, \text { p25: } 0 \\
\text { p75: } 75 \text { ). A vitalidade foi a categoria com } \\
\text { menor amplitude (mediana: } 70 ; \text { p25: } 56,25 \text {; } \\
\text { p75: } 85 \text { ). }\end{array}$ & $\begin{array}{l}\text { O câncer, historicamente, é avaliado } \\
\text { por meio de taxas de sobrevida e cura. } \\
\text { Porém, essa pesquisa busca trazer a } \\
\text { importância de avaliar essa doença a } \\
\text { partir da visão do paciente, mais } \\
\text { especificamente por sua percepção de } \\
\text { qualidade de vida, mostrando como ela } \\
\text { pode ser afetada pela doença e por } \\
\text { outros aspectos paralelos. }\end{array}$ \\
\hline
\end{tabular}


De acordo com os artigos científicos encontrados nota-se que o câncer colorretal possui uma posição de destaque em relação às neoplasias existentes. A incidência deste câncer em pacientes com menos de 40 anos são relativamente baixos e aqueles indivíduos com mais de 50 anos representam cerca de 90,0\%. Um problema óbvio com esta doença são os sintomas silenciosos durante a sua evolução, o que reduz a chance de detecção precoce. Portanto, um prognóstico eficaz está diretamente relacionado ao diagnóstico precoce (Macêdo et al., 2020).

O CCR tem status de problema de saúde pública não só no Brasil, mas também no mundo. Mais da metade dos pacientes com CCR (60-70\%) são diagnosticados com doença avançada, o que leva ao aumento da morbidade e mortalidade. Estratégias de triagem, como teste de sangue oculto nas fezes (PSOF) para a população geral a partir dos 50 anos ou para a população de risco intermediário a partir dos 45 anos, podem efetivamente reduzir a morbidade e a mortalidade (Palmeira et al., 2020; Freitas et al., 2020).

No Brasil, apesar da alta incidência de câncer colorretal, não é disponibilizado nenhum procedimento de rastreamento de pacientes assintomáticos no Sistema Único de Saúde (SUS). Atualmente, tais políticas públicas se limitam ao câncer de mama e câncer do colo do útero. O Ministério da Saúde (MS) atualmente não acredita que a implantação de um programa de rastreamento populacional com câncer colorretal no Brasil seja viável e custo-efetiva, e recomenda apenas uma abordagem individualizada para as situações de risco elevado (Silva et al., 2020).

Os sintomas associados dependerão da localização do câncer e da gravidade do tumor. Os mais comuns nesses pacientes são dispnéia, náusea, perda de apetite, constipação, diarreia, dor, fadiga e insônia. A gravidade de cada caso dependerá do tipo histológico e do estágio em que o câncer se enquadra, afetando a escolha do tipo de tratamento. Apesar dos avanços médicos no tratamento deste câncer, os resultados em pacientes idosos são considerados menos promissores em comparação com pacientes mais jovens (Moura et al.,2020; Aguiar et al., 2020).

O câncer colorretal é uma doença multifatorial relacionada a fatores genéticos e ambientais. Os principais fatores de risco para CCR são: idade acima de 50 anos, obesidade, sedentarismo, tabagismo, alcoolismo, dieta rica em carnes vermelhas ou alimentos processados, pobre em frutas e fibras. Além de uma história familiar positiva de CCR e / ou pólipos adenomatosos, história de doença inflamatória intestinal, diabetes tipo 2 e outros fatores menos comuns (Palmeira et al., 2020; Lima; Villela, 2021).

O desenvolvimento do câncer colorretal inicia-se com a mutação das células epiteliais intestinais, que tem como vantagem a proliferação. O aumento da proliferação origina-se da formação de pólipos adenomatosos benignos, que podem evoluir para a ocorrência de tumores malignos. O tempo evolutivo entre o adenoma e o desenvolvimento do câncer é de cerca de 10 anos. Como outros tipos de câncer, esse tipo de neoplasia pode ser prevenido mantendo o peso adequado, fazendo exercícios e tendo uma dieta saudável, além de não se expor ao tabagismo (Silva et al., 2021).

O estadiamento clínico (I, II, III, IV) é um método para identificar a expansão local de tumores malignos. Quando se referem a estágios iniciais (I e II), podem ter um bom prognóstico. No entanto, os estágios clínicos III e IV são considerados tumores avançados. O objetivo de dividir os casos de câncer é em consequência do fato de haver maior sobrevida quando a doença não se espalhou para outros órgãos (Fagundes, 2020).

Dados epidemiológicos reforçam a importância do rastreamento do câncer, principalmente devido ao aumento de casos de CCR nos últimos 30 anos. Esta realidade é atribuída a fatores ambientais, principalmente relacionados ao estilo de vida (sedentarismo, desnutrição, obesidade, tabagismo) e envelhecimento população. Portanto, a triagem é baseada nos fatores de risco. A idade foi considerada um dos fatores mais importantes. Ao longo dos anos, houve cada vez mais casos de CCR em indivíduos com menos de 50 anos. Portanto, o rastreamento se tornou cada vez mais prematuro. O rastreio deve iniciar aos 45 anos, pois a detecção precoce do CCR pode reduzir significativamente as taxas de mortalidade (De Paula Pires et al., 2021).

O prognóstico do câncer colorretal depende especialmente do estágio patológico no momento do diagnóstico, e a taxa de 
sobrevida em 5 anos varia de $74 \%$ no estágio I a 5,7\% no estágio IV. A cirurgia é considerada o principal tratamento com potencial curativo e deve ser associado à terapia neoadjuvante e / ou adjuvante, como quimioterapia e radioterapia. As estratégias variam de acordo com o estágio e tipo de câncer, desde a ressecção endoscópica até uma cirurgia aberta com ressecções importantes (Paes et al., 2021).

O tratamento do CCR é baseado na manifestação da doença, ou seja, de acordo com a localização e estadiamento do tumor, pode-se utilizar o tratamento cirúrgico, combinado ou não combinado com quimioterapia e radioterapia. A maioria dos pacientes recebe quimioterapia paliativa sistêmica, cujo objetivo clínico é melhorar a qualidade de vida e alcançar a sobrevida (Zanatto et al., 2020).

O câncer sempre foi avaliado de acordo com as taxa de sobrevivência e taxa de cura dos pacientes. Entretanto, estudos têm mostrado a importância da avaliação do câncer por meio da qualidade de vida do paciente, e como este paciente foi afetado pela doença. Além disso, também é importante avaliar os aspectos paralelos ao câncer, como as comorbidades, limitações físicas e emocionais, que também parecem estar relacionados à qualidade de vida e necessitam de auxílio de uma equipe multiprofissional para esses pacientes (Puty et al., 2020).

É necessário refletir sobre os efeitos do câncer colorretal avançado na qualidade de vida relacionada à saúde do paciente e de seus familiares, bem como em seu bem-estar físico, psicológico, social e espiritual. Portanto, deve-se considerar a importância de compreender o indivíduo como um todo e prestar assistência multiprofissional para a melhoria da qualidade de vida desses indivíduos (Fagundes, 2020).

\section{Conclusão}

O câncer colorretal é considerado uma das neoplasias mais prevalentes do mundo. Anteriormente era mais comum em pacientes com idade acima de 50 anos, mas essa realidade tem mudado devido ao estilo de vida da população. É considerado um dos cânceres que mais mata mundialmente em consequência da forma de apresentação da doença. Geralmente essa neoplasia é assintomática e é diagnosticada somente em estágios mais avançados em que o tratamento não é capaz de levar ao processo de cura desses pacientes.

Sabe-se que tratamento dessa neoplasia é realizado através de quimioterapia, radioterapias e em alguns casos é utilizado o procedimento de remoção das partes afetadas pelo câncer através de procedimento cirúrgico. Pacientes estomizados possui uma diminuição da qualidade de vida e é necessário que uma equipe multiprofissional faça o acompanhamento desses pacientes. Infelizmente não há um rastreio desse tipo de câncer que seja oferecido pelo Sistema Único de Saúde (SUS) e por isso o diagnóstico precoce não ocorre com a frequência esperada.

Em virtude dos fatos mencionados, é necessário que sejam desenvolvidas formas de rastreio deste câncer, assim como as realizadas no câncer de mama e de colo do útero, visto que o câncer colorretal é agressivo, possui altas taxas de mortalidade e apresenta uma diminuição significativa da qualidade de vida dos pacientes. É imprescindível também que sejam realizadas campanhas de conscientização sobre essa neoplasia para a população, ressaltando a importância de ter uma vida saudável e do diagnóstico precoce.

\section{Referências}

Aguiar, S., Oliveira, M. M. D, Silva, D. R. M, Mello, C. A. L. D, Calsavara, V. F, \& Curado, M. P (2020). Sobrevivência de pacientes com câncer colorretal em um Centro de Câncer. Arquivos de Gastroenterologia, 57, 172-177.

Bray, F., Ferlay, J., Soerjomataram, I., Siegel, R. L., Torre, L. A., \& Jemal, A. (2018). Global cancer statistics 2018: GLOBOCAN estimates of incidence and mortality worldwide for 36 cancers in 185 countries. CA: a cancer journal for clinicians, 68(6), 394-424.

De Paula Pires, M. E., Mezzomo, D. S., Leite, F. M. M., de Lucena, T. M., Pinheiro, M. J. A., Vargas, L. J., \& Oliveira, M. C. (2021). Rastreamento do Câncer Colorretal: Revisão de literatura. Brazilian Journal of Health Review, 4(2), 6866-6881. 
Fagundes, A. A (2020). Espiritualidade e qualidade de vida relacionada à saúde de pessoas com câncer colorretal avançado (Dissertação de Doutorado, Universidade de São Paulo).

Freitas, B. A. D, Loth, C. A. T, Swarowsky, G. L, Lourenço, G. M, Fillmann, L. S, Fillmann, H. S, \& Padoin, A. V (2020). Obesidade e desenvolvimento de adenoma estão associados como precursores do câncer colorretal? ABCD. Arquivos Brasileiros de Cirurgia Digestiva (São Paulo) , 33 .

Keum, N., \& Giovannucci, E. (2019). Global burden of colorectal cancer: emerging trends, risk factors and prevention strategies. Nature reviews Gastroenterology \& hepatology, 16(12), 713-732.

Lima, M. A. N., \& Villela, D. A. M. (2021). Fatores sociodemográficos e clínicos associados ao tempo para o início do tratamento de câncer de cólon e reto no Brasil, 2006-2015. Cadernos de Saúde Pública, 37, e00214919.

Macêdo, L. M., Cavalcante, V. M. V., Coelho, M. D. M. F., Ramos, S. L. T. C., Correia, D. L., Menezes, T. A. C., \& Rodrigues, A. B. (2020). Percepções de pacientes estomizados com câncer colorretal acerca da qualidade de vida. Rev Rene. 21:e43946.

Mello, M. R. S. P. D., Moura, S. F., Muzi, C. D., \& Guimarães, R. M. (2020). Clinical evaluation and pattern of symptoms in colorectal cancer patients. Arquivos de gastroenterologia, 57, 131-136.

Moura, S. F., de Mello, M. R. S. P., Muzi, C. D., \& Guimarães, R. M. (2020). Padrão Sintomatológico em Pacientes do Câncer Colorretal de acordo com a Idade. Revista Brasileira de Cancerologia, 66(1).

Paes, B. P., Gouveia, A. L. B., de Barros, A. B. S. R., Pereira, A. C. C., do Nascimento, B. P., Velho, G. C. M., \& Trindade, A. V. (2021). Análise das complicações pós-operatórias de câncer colorretal. Brazilian Journal of Development, 7(7), 70884-70896.

Palmeira, I. P., de Souza Guimarães, L., dos Santos, A. K. T., de Andrade, R. L. B., de Almeida Figueiredo, M. B. G., Nunes, M. A. P., \& Lima, S. O. (2020). Evolução comparativa e temporal das tendências de mortalidade por Câncer Colorretal em Sergipe e Nordeste no período de 2008 a 2018 . Brazilian Journal of Health Review, 3(4), 9058-9074.

Puty, T. C., Sarraf, Y. S., Cunha, R. D. P. C., de Carvalho, L. E. W., \& Adami, F. (2020). Perfil da qualidade de vida de pacientes com câncer colorretal estádios III e IV em instituição privada no Estado do Pará: Estudo seccional. Brazilian Journal of Health Review, 3(5), 13403-13411.

Silva, F., Duarte, R. P, Leão, C. C. A, Vissoci, C., Alvarenga, A. L. A. T, Ramis, A. B. S, \& Goulart, A. E. C (2020). Câncer colorretal em pacientes com idade inferior a 50 anos de experiência em cinco anos. Revista do Colégio Brasileiro de Cirurgiões , 47.

Silva, A. L. C, da Silva, L. J, Oliveira, A. S, de Oliveira, R. L, e de Almuinha Ramos, L. G (2021). Cuidados de enfermagem aos pacientes portadores de câncer colorretal. Research, Society and Development, 10 (9), e46910918281-e46910918281.

Zanatto, R. M., Santos, G., Oliveira, J. C., Pracucho, e. M., Nunes, a. J. F., Lopes-Filho, G. J., \& Saad, S. S. (2020). Impacto das mutações KRAS e características clínicas em câncer colorretal. ABCD. Arquivos Brasileiros de Cirurgia Digestiva (São Paulo), 33. 